\title{
HOLDERIAN WEAK INVARIANCE PRINCIPLE FOR STATIONARY MIXING SEQUENCES
}

\author{
DAVIDE GIRAUDO
}

\begin{abstract}
We provide some sufficient mixing conditions on a strictly stationary sequence in order to guarantee the weak invariance principle in Hölder spaces. Strong mixing, $\rho$-mixing conditions are investigated as well as $\tau$-dependent sequences. The main tools are deviation inequalities for mixing sequences.
\end{abstract}

\section{INTRODUCTION}

1.1. Context and notations. Let $\left(X_{j}\right)_{j \geqslant 0}$ be a strictly stationary sequence of real valued random variables with zero mean and finite variance, and for an integer $n \geqslant 1, S_{n}:=\sum_{j=1}^{n} X_{j}$ denotes the $n$-th partial sum. Its variance is denoted by $\sigma_{n}^{2}$. Let us consider the partial sum process

$$
S_{n}^{\mathrm{pl}}(t):=\sum_{j=1}^{[n t]} X_{j}+(n t-[n t]) X_{[n t]+1}, \quad n \geqslant 1, t \in[0,1] .
$$

We are interested in the asymptotic behavior of $\sigma_{n}^{-1} S_{n}^{\mathrm{pl}}(\cdot)$ viewed as a random function in some function spaces.

Notation 1.1. If $T: \Omega \rightarrow \Omega$ is a bi-measurable measure preserving map, we define for $f: \Omega \rightarrow \mathbb{R}$ and a positive integer $n$ the $n$th partial sum $S_{n}(f):=\sum_{j=1}^{n} f \circ T^{j}$ and $\sigma_{n}^{2}(f):=\mathbb{E}\left[S_{n}^{2}(f)\right]-\left(\mathbb{E}\left[S_{n}(f)\right]\right)^{2}$ denotes its variance. We shall also consider $S_{n}^{\mathrm{pl}}(f)$ defined in a similar way as in (1.1), that is,

$$
S_{n}^{\mathrm{pl}}(f, t):=S_{[n t]}(f)+(n t-[n t]) f \circ T^{[n t]+1},
$$

and $W_{n}(f, t):=n^{-1 / 2} S_{n}^{\mathrm{pl}}(f, t)$.

In all the paper, the involved sequences of random variable are assumed to be strictly stationary.

When $\left(X_{j}\right)_{j \geqslant 0}$ is an independent identically distributed sequence, Donsker showed (cf. [6]) that $\left(n^{-1 / 2}\left(\mathbb{E}\left(X_{1}^{2}\right)\right)^{-1 / 2} S_{n}^{\mathrm{pl}}\right)_{n \geqslant 1}$ converges in distribution in the space of continuous functions on the unit interval to a standard Brownian motion $W$. An intensive research has then been performed to extend this result to stationary weakly dependent sequences. We refer the reader to [12] for the main theorems in this area.

In this paper, we rather focus on the convergence in distribution of the partial sum in other function spaces.

Date: October 25, 2018.

2010 Mathematics Subject Classification. 60F05; 60F17.

Key words and phrases. Invariance principle, mixing conditions, strictly stationary process. 
1.2. Hölder spaces. It is well known that standard Brownian motion's paths are almost surely Hölder regular of exponent $\alpha$ for each $\alpha \in(0,1 / 2)$, hence it is natural to consider the random function defined in (1.2) as an element of $\mathcal{H}_{\alpha}[0,1]$ and try to establish its weak convergence to a standard Brownian motion in this function space.

Before stating the results in this direction, let us define for $\alpha \in(0,1)$ the Hölder space $\mathcal{H}_{\alpha}[0,1]$ of functions $x:[0,1] \rightarrow \mathbb{R}$ such that $\sup _{s \neq t}|x(s)-x(t)| /|s-t|^{\alpha}$ is finite. The analogue of the continuity modulus in $C[0,1]$ is $w_{\alpha}$, defined by

$$
w_{\alpha}(x, \delta)=\sup _{0<|t-s|<\delta} \frac{|x(t)-x(s)|}{|t-s|^{\alpha}} .
$$

We then define $\mathcal{H}_{\alpha}^{0}[0,1]$ by $\mathcal{H}_{\alpha}^{0}[0,1]:=\left\{x \in \mathcal{H}_{\alpha}[0,1], \lim _{\delta \rightarrow 0} w_{\alpha}(x, \delta)=0\right\}$. We shall essentially work with the space $\mathcal{H}_{\alpha}^{0}[0,1]$ which, endowed with $\|\cdot\|_{\alpha}: x \mapsto w_{\alpha}(x, 1)+$ $|x(0)|$, is a separable Banach space (while $\mathcal{H}_{\alpha}[0,1]$ is not). Since the canonical embedding $\iota: \mathcal{H}_{\alpha}^{0}[0,1] \rightarrow \mathcal{H}_{\alpha}[0,1]$ is continuous, each convergence in distribution in $\mathcal{H}_{\alpha}^{0}[0,1]$ also takes place in $\mathcal{H}_{\alpha}[0,1]$.

In order to prove such a convergence, we need a tightness criterion. Combining Theorem 14 in [22] in the particular case of the partial sum process (1.2) with Lemma 3.3 in [13], the condition

$$
\forall \varepsilon>0, \quad \lim _{\delta \rightarrow 0} \limsup _{n \rightarrow \infty} \frac{1}{\delta} \sum_{j=1}^{\log [n \delta]} 2^{j} \mu\left\{\max _{1 \leqslant k \leqslant[n \delta] 2^{-j}}\left|S_{k}(f)\right|>\frac{\sigma_{n}}{n^{\alpha}} \varepsilon\left([n \delta] 2^{-j}\right)^{\alpha}\right\}=0
$$

(where log denotes the binary logarithm) is sufficient for tightness of the sequence $\left(\sigma_{n}^{-1}(f) S_{n}^{\mathrm{pl}}(f)\right)_{n \geqslant 1}$ in $\mathcal{H}_{\alpha}^{0}[0,1]$.

In the particular case of linear variance (that is, $\sigma_{n}^{2} \sim c n$ as $n \rightarrow \infty$ for some constant $c$ ), we will normalize by $\sqrt{n}$. Using the change of indexes $k=\log [n \delta]-j$ (so that $\left.2^{-j}=2^{k} /[n \delta]\right)$, this leads to the following tightness criterion for $\left(W_{n}(f)\right)_{n \geqslant 1}$ in $\mathcal{H}_{\alpha}^{0}[0,1]$ :

$$
\forall \varepsilon>0, \quad \lim _{\delta \rightarrow 0} \limsup _{n \rightarrow \infty} n \sum_{k=1}^{\log [n \delta]} 2^{-k} \mu\left\{\max _{1 \leqslant i \leqslant 2^{k}}\left|S_{i}(f)\right|>\varepsilon 2^{k \alpha} n^{1 / p}\right\}=0,
$$

where $\alpha=1 / 2-1 / p$.

As mentioned before, the random function defined in (1.1) can be viewed as an element of $\mathcal{H}_{\alpha}[0,1], \alpha \in(0,1 / 2)$ and we can try to establish the weak convergence of the sequence $\left(\sigma_{n}^{-1} S_{n}^{\mathrm{pl}}(f)\right)_{n \geqslant 1}$ to a standard Brownian motion in this function space. To the best of our knowledge, it seems that the study of this kind of convergence was not as intensive as in the space of continuous functions or the Skorohod space. The first result in this direction was established by Lamperti in [11]: if $\left(X_{j}\right)_{j \geqslant 0}$ is an i.i.d. sequence with $\mathbb{E}\left[X_{0}\right]=0, \mathbb{E}\left[X_{0}^{2}\right]=1$ and $\mathbb{E}\left|X_{0}\right|^{p}$ is finite, then the sequence $\left(n^{-1 / 2} S_{n}^{\mathrm{pl}}\right)_{n \geqslant 1}$ converges to a standard Brownian motion in $\mathcal{H}_{\gamma}^{0}[0,1]$ for each $\gamma<1 / 2-1 / p$. Later, Račkauskas and Suquet improved this result (cf. [15]), showing that for an i.i.d. zero mean sequence, a necessary and sufficient condition to obtain the invariance principle in $\mathcal{H}_{1 / 2-1 / p}^{0}[0,1]$ is $\lim _{t \rightarrow \infty} t^{p} \mu\left\{\left|X_{0}\right|>t\right\}=0$ (in [16] they considered the case of more general Hölder spaces, where the role of $t \mapsto t^{\alpha}$ is played by $t \mapsto t^{\alpha} L(t)$ with some conditions on $L$ ). 
Thus, establishing the weak convergence of the partial sum process in Hölder spaces requires, even in the independent case, finite moment of order greater than 2 and the moment condition depends on the exponent of the considered Hölder space. It is a natural question to ask about generalizations of the result by Račkauskas and Suquet for dependent sequences. In this paper, we focus on strictly stationary sequences satisfying some mixing conditions (see next section).

1.3. Mixing conditions. We present the mixing conditions involved in the paper.

Let $\mathcal{A}$ and $\mathcal{B}$ be two sub- $\sigma$-algebras of $\mathcal{F}$, where $(\Omega, \mathcal{F}, \mu)$ is a probability space. We define the $\alpha$-mixing coefficients as introduced by Rosenblatt in [18]:

$$
\alpha(\mathcal{A}, \mathcal{B}):=\sup \{|\mu(A \cap B)-\mu(A) \mu(B)|, A \in \mathcal{A}, B \in \mathcal{B}\} .
$$

The $\rho$-mixing coefficients were introduced by Hirschfeld [9] and are defined by

$$
\rho(\mathcal{A}, \mathcal{B}):=\sup \left\{|\operatorname{Corr}(f, g)|, f \in \mathbb{L}^{2}(\mathcal{A}), g \in \mathbb{L}^{2}(\mathcal{B}), f \neq 0, g \neq 0\right\},
$$

where $\operatorname{Corr}(f, g):=[\mathbb{E}(f g)-\mathbb{E}(f) \mathbb{E}(g)]\left[\|f-\mathbb{E}(f)\|_{\mathbb{L}^{2}}\|g-\mathbb{E}(g)\|_{\mathbb{L}^{2}}\right]^{-1}$.

The coefficients are related by the inequalities

$$
4 \alpha(\mathcal{A}, \mathcal{B}) \leqslant \rho(\mathcal{A}, \mathcal{B}) .
$$

For a strictly stationary sequence $\left(X_{k}\right)_{k \in \mathbb{Z}}$ and $n \geqslant 0$ we define $\alpha_{X}(n)=\alpha(n)=$ $\alpha\left(\mathcal{F}_{-\infty}^{0}, \mathcal{F}_{n}^{\infty}\right)$ where $\mathcal{F}_{u}^{v}$ is the $\sigma$-algebra generated by $X_{k}$ with $u \leqslant k \leqslant v$ (if $u=-\infty$ or $v=\infty$, the corresponding inequality is strict). In the same way we define coefficients $\rho_{X}(n)$.

When there will be no ambiguity, we shall simply write $\alpha(n)$ and $\rho(n)$. We say that the sequence $\left(X_{k}\right)_{k \in \mathbb{Z}}$ is $\alpha$-mixing if $\lim _{n \rightarrow+\infty} \alpha(n)=0$, and similarily we define $\rho$-mixing sequences.

$\alpha$-mixing sequences were considered in the mentioned references, while $\rho$-mixing sequences first appeared in [10. Inequality (1.5) translated in terms of mixing coefficients of a sequence states that for each positive integer $n$,

$$
4 \alpha(n) \leqslant \rho(n) .
$$

In particular, a $\rho$-mixing sequence is $\alpha$-mixing.

1.4. $\tau$-dependence coefficient. In order to define the $\tau$-dependence coefficients of a stationary sequence, we first need a result about conditional probability (see Theorem 33.3 of [1]).

Lemma 1.2. Let $(\Omega, \mathcal{F}, \mu)$ be a probability space, $\mathcal{M}$ a sub- $\sigma$-algebra of $\mathcal{F}$ and $X$ a real-valued random variable with distribution $\mu_{X}$. There exists a function $\mu_{X \mid \mathcal{M}}$ from $\mathcal{B}(\mathbb{R}) \times \Omega$ to $[0,1]$ such that

(1) For any $\omega \in \Omega, \mu_{X \mid \mathcal{M}}(\cdot, \omega)$ is a probability measure on $\mathcal{B}(\mathbb{R})$.

(2) For any $A \in \mathcal{B}(\mathbb{R}), \mu_{X \mid \mathcal{M}}(A, \cdot)$ is a version of $\mathbb{E}\left[\mathbf{1}_{\{X \in A\}} \mid \mathcal{M}\right]$.

We now introduce the $\tau$-dependence coefficients as in $\left[4\right.$. We denote by $\Lambda_{1}(\mathbb{R})$ the collection of 1 -Lipschitz functions from $\mathbb{R}$ to $\mathbb{R}$ and define the quantity

$$
W\left(\mu_{X \mid \mathcal{M}}\right):=\sup \left\{\left|\int f(x) \mu_{X \mid \mathcal{M}}(\mathrm{d} x)-\int f(x) \mu_{X}(\mathrm{~d} x)\right|, f \in \Lambda_{1}(\mathbb{R})\right\} .
$$

For an integrable random variable $X$ and a sub- $\sigma$-algebra $\mathcal{M}$, the coefficient $\tau$ is defined by

$$
\tau(\mathcal{M}, X)=\left\|{ }_{3} W\left(\mu_{X \mid \mathcal{M}}\right)\right\|_{1} .
$$


This definition can be extended to random variables with values in finite dimensional vector spaces. If $d$ is a positive integer, we endow $\mathbb{R}^{d}$ with the norm $\|x-y\|:=$ $\sum_{j=1}^{n}\left|x_{j}-y_{j}\right|$ and define $\Lambda_{1}\left(\mathbb{R}^{d}\right)$ as the set of 1-Lipschitz functions from $\mathbb{R}^{d}$ to $\mathbb{R}$.

Definition 1.3. Let $(\Omega, \mathcal{F}, \mu)$ be a probability space, $\mathcal{M}$ a sub- $\sigma$-algebra of $\mathcal{F}$ and $X$ an $\mathbb{R}^{d}$-valued random variable. We define

$$
\tau(\mathcal{M}, X):=\sup \left\{\tau(\mathcal{M}, f(X)), f \in \Lambda_{1}(E)\right\} .
$$

We can now introduce the $\tau$-mixing coefficient for a sequence of real-valued random variables.

Definition 1.4. Let $\left(X_{i}\right)_{i \geqslant 1}$ be a sequence of random variables and $\left(\mathcal{M}_{i}\right)_{i \geqslant 1}$ a sequence of sub- $\sigma$-algebras of $\mathcal{F}$. For any positive integer $k$, define

$$
\tau(i):=\max _{p, l \geqslant 1} \frac{1}{l} \sup \left\{\tau\left(\mathcal{M}_{p},\left(X_{j_{1}}, \ldots, X_{j_{l}}\right)\right), p+i \leqslant j_{1}<\cdots<j_{l}\right\} .
$$

In the sequel, we shall focus on the case $\mathcal{M}_{i}:=\sigma\left(X_{k}, k \leqslant i\right)$.

Notation 1.5. Let $X: \Omega \rightarrow \mathbb{R}$ be a random variable. We denote $Q_{X}(\cdot)$ the inverse function defined by $Q_{X}(u):=\inf \{t, \mu\{|X|>t\} \leqslant u\}$. If $\left(f \circ T^{j}\right)_{j \geqslant 0}$ is a strictly stationary sequence and $(\alpha(n))_{n \geqslant 1}$ is its sequence of $\alpha$-mixing coefficients, we denote by $\alpha^{-1}(u)$ the number of indices $n$ for which $\alpha(n) \geqslant u$. More generally, if $\left(\delta_{i}\right)_{i \geqslant 0}$ is a non-increasing sequence of non-negative numbers, we define $\delta^{-1}(u):=\inf \left\{k \in \mathbb{N}, \delta_{k} \leqslant u\right\}$.

We can compare the $\tau$-dependence coefficient with the $\alpha$-mixing coefficients. The following is a simplified version of Lemma 7 of [2].

Lemma 1.6. Let $\left(f \circ T^{j}\right)_{j \geqslant 0}$ be a strictly stationary sequence. Then for each integer $i$, the following inequality holds:

$$
\tau(i) \leqslant 2 \int_{0}^{2 \alpha(i)} Q_{f}(u) \mathrm{d} u .
$$

In [2], "Application 1: causal linear processes" (p. 871), Dedecker and Prieur provide an example of a process whose $\tau$-dependence coefficients converge to 0 as fast as $2^{-i}$ but $\alpha_{i}=1 / 4$ for each positive integer $i$.

\section{MAIN RESUltS}

2.1. Mixing sequences. In this subsection, we give sufficient mixing conditions which guarantee the convergence of the sequence $\left(W_{n}(f)\right)_{n \geqslant 1}$ to a Brownian motion in the space $\mathcal{H}_{1 / 2-1 / p}^{0}[0,1], p>2$.

We refer the reader to Notations 1.5 and A.2

Theorem 2.1. Let $p>2$ and let $\left(f \circ T^{j}\right)_{j \geqslant 0}$ be a strictly stationary centered sequence such that

$$
\lim _{t \rightarrow \infty} t^{p-1} \int_{0}^{1} Q_{f}(u) \mathbf{1}\left\{\left((\tau / 2)^{-1} \circ G_{f}^{-1}\right)(u) Q_{f}(u)>t\right\} \mathrm{d} u=0 .
$$

Then

$$
W_{n}(f) \rightarrow \sigma(f) W \text { in distribution in } \mathcal{H}_{1 / 2-1 / p}^{0}[0,1] \text {, }
$$

where $\sigma^{2}(f)=\operatorname{Var}(f)+2 \sum_{k=1}^{\infty} \operatorname{Cov}\left(f, f \circ T^{k}\right)$. 
Using the comparison between $\alpha$ and $\tau$, we can deduce a condition in the spirit of that of Doukhan, Massart and Rio (see [7]). One can also derive it from the tightness criterion (1.4) and Theorem 6.2 of [17].

Corollary 2.2. Let $p>2$ and let $\left(f \circ T^{j}\right)_{j \geqslant 0}$ be a strictly stationary centered sequence such that

$$
\lim _{t \rightarrow \infty} t^{p-1} \int_{0}^{1} Q_{f}(u) \mathbf{1}\left\{\alpha^{-1}(u) Q_{f}(u)>t\right\} \mathrm{d} u=0 .
$$

Then

$$
W_{n}(f) \rightarrow \sigma(f) W \text { in distribution in } \mathcal{H}_{1 / 2-1 / p}^{0}[0,1] \text {, }
$$

where $\sigma^{2}(f)=\lim _{n \rightarrow \infty} \sigma_{n}^{2}(f) / n$.

Remark 2.3. Assume that the sequence $\left(f \circ T^{j}\right)_{j \geqslant 0}$ is independent and that $t^{p} \mu\{|f|>t\} \rightarrow$ 0 . Then the condition of Theorem 2.1 is satisfied. Indeed, since $Q_{f}(U)$ is distributed as $|f|$ if $U$ is uniformly distributed on $[0,1]$, both conditions (2.2) and (2.4) read

$$
\lim _{t \rightarrow \infty} t^{p-1} \mathbb{E}[|f| \mathbf{1}\{|f|>t\}]=0 .
$$

As

$$
\begin{aligned}
t^{p-1} \mathbb{E}[|f| \mathbf{1}\{|f|>t\}] & =t^{p-1} \int_{0}^{\infty} \mu\{|f|>\max \{u, t\}\} \mathrm{d} u \\
& =t^{p} \mu\{|f|>t\}+t^{p-1} \int_{t}^{\infty} \mu\{|f|>u\} \mathrm{d} u \\
& \leqslant t^{p} \mu\{|f|>t\}+\sup _{s \geqslant t} s^{p} \mu\{|f|>s\} /(p-1),
\end{aligned}
$$

condition (2.5) is satisfied hence we can derive the result by Račkauskas and Suquet in the i.i.d. case from Theorem 2.1, This contrasts with Theorem 17 of [8], from which we can only deduce the result by Lamperti (cf. [11]) in the i.i.d. case.

Remark 2.4. Assume that $Q_{f}(u) \leqslant C u^{-1 / a}$ for some $a>p$ (this is the case if $f$ admits a finite weak moment of order $a)$. If $\alpha(k)=o\left(k^{-a(p-1) /(a-p)}\right)$ or $\tau(k)=o\left(k^{-(a-1)(p-1) /(a-p)}\right)$, then condition (2.1) holds. If $f$ is bounded, these sufficient conditions can be weakened respectively to $\alpha(k)=o\left(k^{-(p-1)}\right)$ and $\tau(k)=$ $o\left(k^{-(p-1)}\right)$.

We conclude this subsection by a result on $\rho$-mixing sequences.

Theorem 2.5. Let $p>2$ and let $\left(f \circ T^{j}\right)_{j \geqslant 0}$ be a strictly stationary centered sequence such that $t^{p} \mu\{|f|>t\} \rightarrow 0$ as $t \rightarrow+\infty$ and $\sum_{i=0}^{\infty} \rho\left(2^{i}\right)<\infty$. Then

$$
W_{n}(f) \rightarrow \sigma(f) W \text { in distribution in } \mathcal{H}_{1 / 2-1 / p}^{0}[0,1] \text {, }
$$

where $\sigma^{2}(f)=\lim _{n \rightarrow \infty} \sigma_{n}^{2}(f) / n$.

2.2. A counter-example. In this subsection, we show that boundedness of the sequence of $p$ th moments of the normalized partial sums is not enough to guarantee tightness in $\mathcal{H}_{1 / 2-1 / p}[0,1]$.

Theorem 2.6. Let $p>2$. There exists a strictly stationary sequence $\left(f \circ T^{j}\right)_{j \geqslant 0}$ such that

- the finite dimensional distributions of $\left(W_{n}(f)\right)_{n \geqslant 1}$ converge to those of a standard Brownian motion,

- the sequence $\left(\mathbb{E}\left|S_{n}(f)\right|^{p} / n^{p / 2}\right)_{n \geqslant 1}$ is bounded and 
- the process $\left(W_{n}(f)\right)_{n \geqslant 1}$ is not tight in $\mathcal{H}_{1 / 2-1 / p}[0,1]$.

Remark 2.7. The constructed process has no reason to be $\alpha$-mixing. However, this proves that in general, establishing tightness in $\mathcal{H}_{1 / 2-1 / p}^{0}[0,1]$ of $\left(W_{n}(f)\right)_{n \geqslant 1}$ cannot be done by proving boundedness in $\mathbb{L}^{p}$ of the sequence $\left(W_{n}(f)\right)_{n \geqslant 1}$. Thus other methods need to be used.

Let us recall that a sequence $\left(c_{n}\right)_{n \geqslant 1}$ is slowly varying if there exists a continuous function $h: \mathbb{R}_{+}^{*} \rightarrow \mathbb{R}_{+}^{*}$ such that $c_{n}=h(n)$ for each positive integer $n$ and for each positive $x, \lim _{t \rightarrow \infty} h(t x) / h(t)=1$.

Remark 2.8. If $p>2$ and $\left(f \circ T^{j}\right)_{j \geqslant 0}$ is a strictly stationary centered sequence such that the finite dimensional distributions of $\left(\sigma_{n}^{-1} S_{n}^{\mathrm{pl}}(f)\right)_{n \geqslant 1}$ converge to those of a standard Brownian motion, the sequence $\left(\sigma_{n}^{2}(f) / n\right)_{n \geqslant 1}$ is slowly varying, and the sequence $\left(\mathbb{E}\left|S_{n}(f)\right|^{p} / \sigma_{n}^{p}\right)_{n \geqslant 1}$ is bounded, then for each $\gamma<1 / 2-1 / p$ the sequence $\left(\sigma_{n}^{-1} S_{n}^{\mathrm{pl}}(f)\right)_{n \geqslant 1}$ converges in distribution in $\mathcal{H}_{\alpha}^{0}[0,1]$ to a standard Brownian motion. This can be seen using tightness criterion (1.3), Markov's inequality and boundedness in $\mathbb{L}^{p}$ of $\left(\sigma_{n}^{-1} \max _{1 \leqslant j \leqslant n}\left|S_{j}(f)\right|\right)_{n \geqslant 1}$ (by Serfling arguments, see [19]).

\section{Proofs}

Proof of Theorem 2.1. Notice that (2.1) implies finiteness of $\int_{0}^{1} Q_{f}^{2}(u)(\tau / 2)^{-1}$ 。 $G_{f}^{-1}(u) \mathrm{d} u$, hence condition (5.5) in [2]. This implies the convergence of $\left(\sigma_{n}^{2}(f) / n\right)_{n \geqslant 1}$ to $\sigma(f)$. Since $\theta(k)$ is smaller than $\tau(k)$, Corollary 1 in [3] shows that the function $f$ satisfies the projective criterion by Dedecker and Rio (see [5]), from which the convergence of the finite dimensional distributions follows. It remains to check tightness of $\left(W_{n}(f)\right)_{n \geqslant 1}$ in $\mathcal{H}_{1 / 2-1 / p}^{0}[0,1]$. We shall check that (1.4) is satisfied. To this aim, we apply Theorem $\AA .3$ for each $k \in\{1, \ldots, \log [n \delta]\}$ with some $r>p$, $N:=2^{k}$ and $\lambda:=\varepsilon 2^{k \alpha} n^{1 / p}$. This gives

$$
\begin{gathered}
n \sum_{k=1}^{\log [n \delta]} 2^{-k} \mu\left\{\max _{1 \leqslant i \leqslant 2^{k}}\left|S_{i}(f)\right|>5 \varepsilon 2^{k \alpha} n^{1 / p}\right\} \leqslant n \sum_{k=1}^{\log [n \delta]} 2^{-k} 4 r^{r / 2} s_{2^{k}}(f)^{r}\left(\varepsilon 2^{k \alpha} n^{1 / p}\right)^{-r}+ \\
+n \sum_{k=1}^{\log [n \delta]}\left(\varepsilon 2^{k \alpha} n^{1 / p}\right)^{-1} \int_{0}^{1} Q(u) \mathbf{1}\left\{R(u) \geqslant \varepsilon 2^{k \alpha} n^{1 / p} / r\right\} \mathrm{d} u .
\end{gathered}
$$

By (2.1), the quantity $C:=\int_{0}^{\|f\|_{1}}(\tau / 2)^{-1}(u) Q_{f} \circ G_{f}(u) \mathrm{d} u$ is finite. In view of (A.3), we thus have

$$
\begin{gathered}
n \sum_{k=1}^{\log [n \delta]} 2^{-k} \mu\left\{\max _{1 \leqslant i \leqslant 2^{k}}\left|S_{i}(f)\right|>5 \varepsilon 2^{k \alpha} n^{1 / p}\right\} \leqslant 4 \cdot(4 r)^{r / 2} C^{r / 2} n \sum_{k=1}^{\log [n \delta]} 2^{-k} 2^{k r / 2}\left(\varepsilon 2^{k \alpha} n^{1 / p}\right)^{-r}+ \\
+n^{1-1 / p} \sum_{k=1}^{\log [n \delta]}\left(\varepsilon 2^{k \alpha}\right)^{-1} \int_{0}^{1} Q(u) \mathbf{1}\left\{R(u) \geqslant \varepsilon 2^{k \alpha} n^{1 / p} / r\right\} \mathrm{d} u=:(I)+(I I) .
\end{gathered}
$$

A simple computation shows that

$$
(I) \leqslant \underset{6}{K(r, p, \varepsilon) \delta^{r / p-1},}
$$


and for the second term, we have the upper bound

$$
(I I) \leqslant K(\alpha, \varepsilon) n^{(p-1) / p} \int_{0}^{1} Q(u) \mathbf{1}\left\{(\tau / 2)^{-1} \circ G_{f}^{-1}(u) Q(u) \geqslant \varepsilon n^{1 / p} / r\right\} \mathrm{d} u .
$$

Since $r>p$, the condition (1.4) is satisfied in view of (3.1), (3.2), (3.3) and (2.1).

Proof of Corollary 2.2. It suffices to check that condition (2.3) implies (2.1). Notice that by (1.9), we have for a fixed $v$,

$$
\inf \{i \mid \tau(i) / 2 \leqslant v\} \leqslant \inf \left\{i \mid G^{-1}(2(\alpha(i))) \leqslant v\right\}=\inf \{i \mid \alpha(i) \leqslant G(v) / 2\}
$$
hence $(\tau / 2)^{-1}(v) \leqslant \alpha^{-1}(G(v) / 2)$. Taking $v=G^{-1}(u)$ for a fixed $u$, we get

$$
(\tau / 2)^{-1} \circ G^{-1}(u) \leqslant \alpha^{-1}(u / 2) .
$$

Since the function $u \mapsto \alpha^{-1}(u)$ is non-increasing, the inclusion

$$
\left\{(\tau / 2)^{-1} \circ G^{-1}(u) Q_{f}(u)>t\right\} \subset\left\{\alpha^{-1}(u / 2) Q_{f}(u / 2)>t\right\} .
$$

takes place. As a consequence, we obtain

$$
\begin{aligned}
& t^{p-1} \int_{0}^{1} Q_{f}(u) \mathbf{1}\left\{\left((\tau / 2)^{-1} \circ G_{f}^{-1}\right)(u) Q_{f}(u)>t\right\} \mathrm{d} u \leqslant \\
& \leqslant t^{p-1} \int_{0}^{1} Q_{f}(u / 2) \mathbf{1}\left\{\alpha^{-1}(u / 2) Q_{f}(u / 2)>t\right\} \mathrm{d} u
\end{aligned}
$$

which concludes the proof of Corollary 2.2 .

Proof of Theorem 2.5. Theorem 4.1. of [14 guarantees the existence of the limit of the sequence $\left(\sigma_{n}^{2} / n\right)_{n \geqslant 1}$ and [20] gives the convergence of the finite dimensional distributions. Therefore, the proof will be finished if we check the convergence (1.4). We apply for Theorem A.4 for each $1 \leqslant k \leqslant \log [n \delta]$ with a $q>p, N:=2^{k}$ $x:=\varepsilon 2^{k \alpha} n^{1 / p}$ and $A:=2^{k \alpha} n^{1 / p} \eta$, where $\eta$ is fixed (notice that since

$$
\mathbb{E}[|f| \mathbf{1}\{|f| \geqslant A\}]=A \mu\{|f| \geqslant A\}+\int_{A}^{+\infty} \mu\{|f| \geqslant t\} \mathrm{d} t \leqslant C(p, f) A^{1-p},
$$

we have for $n \geqslant n(\eta, p, \varepsilon, f)$ and $1 \leqslant k \leqslant \log [n \delta]$,

$$
2 \cdot 2^{k} \cdot \mathbb{E}[|f| \mathbf{1}\{|f| \geqslant A\}] \leqslant 2 C(p, f)\left(\eta 2^{k \alpha} n^{1 / p}\right)^{1-p} \leqslant \varepsilon 2^{k \alpha} n^{1 / p}=x,
$$

hence (A.5) is satisfied). This yields

$$
\begin{aligned}
& n \sum_{k=1}^{\log [n \delta]} 2^{-k} \mu\left\{\max _{1 \leqslant i \leqslant 2^{k}}\left|S_{i}(f)\right| \geqslant \varepsilon 2^{k \alpha} n^{1 / p}\right\} \leqslant n \sum_{k=1}^{\log [n \delta]} \mu\left\{|f| \geqslant 2^{k \alpha} n^{1 / p}\right\}+ \\
& \quad+K \exp \left(K \sum_{i=0}^{\infty} \rho\left(2^{i}\right)\right) n \sum_{k=1}^{\log [n \delta]} 2^{-k} 2^{k q / 2}\left(\varepsilon 2^{k \alpha} n^{1 / p}\right)^{-q}\|f\|_{2}^{q}+ \\
& \quad+K n \sum_{k=1}^{\log [n \delta]} \exp \left(K \sum_{i=0}^{k} \rho^{2 / q}\left(2^{i}\right)\right)\left(\varepsilon 2^{k \alpha} n^{1 / p}\right)^{-q}\left\|f \mathbf{1}\left\{|f| \leqslant \eta 2^{k \alpha} n^{1 / p}\right\}\right\|_{q}^{q}
\end{aligned}
$$

Since for some constant $C$ depending only on $f$ and $p$, the bound

$$
\left\|f \mathbf{1}\left\{|f| \leqslant \eta 2^{k \alpha} n^{1 / p}\right\}\right\|_{q}^{q} \leqslant C\left(\eta 2^{k \alpha} n^{1 / p}\right)^{q-p}
$$


is valid, we derive from (3.9) the inequality

$$
\begin{aligned}
& n \sum_{k=1}^{\log [n \delta]} 2^{-k} \mu\left\{\max _{1 \leqslant i \leqslant 2^{k}}\left|S_{i}(f)\right| \geqslant\right.\left.\varepsilon 2^{k \alpha} n^{1 / p}\right\} \leqslant \varepsilon^{-p} \eta^{-p} \sup _{t \geqslant n^{1 / p}} t^{p} \mu\{|f| \geqslant t\} \sum_{k=1}^{\infty} 2^{-k p \alpha}+ \\
&+K \exp \left(K \sum_{i=0}^{\infty} \rho\left(2^{i}\right)\right) \delta^{q / p-1} \cdot \frac{1}{2^{q / p}-1} \varepsilon^{-q}\|f\|_{2}^{q}+ \\
&+K C \varepsilon^{-q} \eta^{q-p} \sum_{k=1}^{\log [n \delta]} \exp \left(K \sum_{i=0}^{k} \rho^{2 / q}\left(2^{i}\right)\right) 2^{-k p \alpha}
\end{aligned}
$$

Since $\lim _{t \rightarrow \infty} t^{p} \mu\{|f|>t\}=0$, we obtain for each $\delta$ and $\eta$

$$
\begin{gathered}
\limsup _{n \rightarrow \infty} n \sum_{k=1}^{\log [n \delta]} 2^{-k} \mu\left\{\max _{1 \leqslant i \leqslant 2^{k}}\left|S_{i}(f)\right|>\varepsilon 2^{k \alpha} n^{1 / p}\right\} \leqslant K \exp \left(K \sum_{i=0}^{\infty} \rho\left(2^{i}\right)\right)(2 \delta)^{q / p-1} \varepsilon^{-q}\|f\|_{2}^{q}+ \\
+K C \varepsilon^{-q} \eta^{q-p} \sum_{k=1}^{\infty} \exp \left(K \sum_{i=0}^{k} \rho^{2 / q}\left(2^{i}\right)\right) 2^{-k p \alpha}
\end{gathered}
$$

from which (1.4) follows (the convergence of the last series is ensured by the ratio test and the convergence to 0 of $\left.\rho^{2 / q}\left(2^{k}\right)\right)$.

Proof of Theorem [2.6. We assume that $(\Omega, \mathcal{F}, \mu, T)$ is a non-atomic invertible measure preserving system. We shall first construct a function $g$ such that:

(1) the sequence $\left(\mathbb{E}\left|S_{n}(g-g \circ T)\right|^{p} / n^{p / 2}\right)_{n \geqslant 1}$ is bounded and

(2) the process $\left(W_{n}(g-g \circ T)\right)_{n \geqslant 1}$ is not tight in $\mathcal{H}_{1 / 2-1 / p}^{0}[0,1]$.

We then consider $f:=m+g-g \circ T$, where $m$ is such that $\left(m \circ T^{j}\right)_{j \geqslant 0}$ is a martingale difference sequence with $m \in \mathbb{L}^{p}$ and $m \neq 0$. This will guarantee the convergence of the finite dimensional distributions of $\left(W_{n}(f)\right)_{n \geqslant 1}$ to those of a scalar multiple of a standard Brownian motion, and Burkholder's inequality ensures boundedness of the sequence $\left(\mathbb{E}\left|S_{n}(f)\right|^{p} / n^{p / 2}\right)_{n \geqslant 1}$. We use a construction similar to that given in [23. Let us consider two increasing sequences of integer $\left(K_{l}\right)_{l \geqslant 1}$ and $\left(N_{l}\right)_{l \geqslant 1}$ satisfying for each $l \geqslant 2$ :

$$
\begin{array}{r}
\lim _{l \rightarrow+\infty} N_{l} \sum_{l^{\prime}>l} K_{l^{\prime}} / N_{l^{\prime}}=0 ; \\
4 N_{l}^{-1 / p} \cdot l \cdot N_{l-1}<1 ; \\
\sum_{i=1}^{l} K_{i}^{1 / 2} \leqslant K_{l+1}^{1 / 2} ; \\
\sum_{l=1}^{+\infty} \frac{K_{l}}{K_{l+1}^{1 / 2}}<\infty .
\end{array}
$$

We also assume that $4 K_{l} \leqslant N_{l}$ for each $l$.

Let us fix an integer $l$. Using Rokhlin's lemma, we can find a set $A_{l} \in \mathcal{F}$ such that the set $T^{i} A_{l}, 0 \leqslant i \leqslant N_{l}-1$ are pairwise disjoint and $\mu\left(\bigcup_{i=0}^{N_{l}-1} T^{i} A_{l}\right) \geqslant 1 / 2$. 
We define

$$
\begin{gathered}
h_{l}:=\frac{N_{l}^{1 / p}}{K_{l}^{1 / 2+1 / p}} \cdot \mathbf{1}\left(\bigcup_{j=1}^{K_{l}} T^{N_{l}-j} A_{l}\right) ; \\
g_{l}:=\sum_{j=0}^{K_{l}-1} h_{l} \circ T^{j}=\frac{N_{l}^{1 / p}}{K_{l}^{1 / 2+1 / p}}\left(\sum_{j=1}^{K_{l}} j \mathbf{1}\left(T^{N_{l}-j} A_{l}\right)+\sum_{j=K_{l}+1}^{2 K_{l}-1}\left(2 K_{l}-j\right) \mathbf{1}\left(T^{N_{l}-j} A_{l}\right)\right) ; \\
g:=\sum_{l=1}^{+\infty} g_{l} .
\end{gathered}
$$

Assume that $\omega \in A_{l}$ and $N_{l}-K_{l} \leqslant i \leqslant N_{l}-1$. Then $g_{l} \circ T^{i}(\omega)=N_{l}-i$. Consequently, for $i, i^{\prime} \in\left\{N_{k}-K_{l}, \ldots, N_{l}-1\right\}$,

$$
\left|g_{l} \circ T^{i}-g_{l} \circ T^{i^{\prime}}\right| \geqslant \frac{N_{l}^{1 / p}}{K_{l}^{1 / 2+1 / p}}\left|i^{\prime}-i\right| \mathbf{1}\left(A_{l}\right) .
$$

Applying $U^{-k}$ on both sides of the previous inequality for $0 \leqslant k \leqslant N_{l}-K_{l}$ and taking the maximum over these $k$, we obtain

$$
\max _{0 \leqslant k \leqslant N_{l}-K_{l}}\left|g_{l} \circ T^{i-k}-g_{l} \circ T^{i^{\prime}-k}\right| \geqslant\left|i^{\prime}-i\right| \frac{N_{l}^{1 / p}}{K_{l}^{1 / 2+1 / p}} \mathbf{1}\left(\bigcup_{k=0}^{N_{k}-K_{l}} T^{k}\left(A_{l}\right)\right) .
$$

This implies

$$
\frac{1}{N_{l}^{1 / p}} \max _{1 \leqslant i<i^{\prime} \leqslant N_{l}} \frac{\left|g_{l} \circ T^{i^{\prime}}-g_{l} \circ T^{i}\right|}{\left(i^{\prime}-i\right)^{1 / 2-1 / p}} \geqslant \mathbf{1}\left(\bigcup_{k=0}^{N_{k}-K_{l}} T^{k}\left(A_{l}\right)\right) .
$$

For $l^{\prime}<l$, noticing that $\left|g_{l^{\prime}}\right| \leqslant N_{l^{\prime}}^{1 / p} K_{l^{\prime}}^{1 / 2-1 / p}$, the following inequality takes place:

$$
\max _{1 \leqslant i<j \leqslant N_{l}}\left|g_{l^{\prime}} \circ T^{j}-g_{l^{\prime}} \circ T^{i}\right| \leqslant 2 N_{l^{\prime}}^{1 / p} K_{l^{\prime}}^{1-1 / p},
$$

therefore,

$$
\begin{aligned}
\frac{1}{N_{l}^{1 / p}} \max _{1 \leqslant i<i^{\prime} \leqslant N_{l}} \frac{\left|\sum_{l^{\prime}<l} g_{l^{\prime}} \circ T^{i^{\prime}}-\sum_{l^{\prime}<l} g_{l^{\prime}} \circ T^{i}\right|}{\left(i^{\prime}-i\right)^{1 / 2-1 / p}} \leqslant \\
\quad \leqslant \frac{1}{N_{l}^{1 / p}} \sum_{l^{\prime}<l} 2 N_{l^{\prime}}^{1 / p} K_{l^{\prime}}^{1-1 / p} \leqslant 2 N_{l}^{-1 / p} l N_{l-1} .
\end{aligned}
$$

By condition (3.12), we conclude that

$$
\frac{1}{N_{l}^{1 / p}} \max _{1 \leqslant i<i^{\prime} \leqslant N_{l}} \frac{\left|\sum_{l^{\prime}<l} g_{l^{\prime}} \circ T^{i^{\prime}}-\sum_{l^{\prime}<l} g_{l^{\prime}} \circ T^{i}\right|}{\left(i^{\prime}-i\right)^{1 / 2-1 / p}} \leqslant 1 / 2 .
$$


Moreover, notice that

$$
\begin{aligned}
\mu\left\{N_{l}^{-1 / p} \max _{1 \leqslant i<i^{\prime} \leqslant N_{l}} \frac{\left|\sum_{l^{\prime}>l} g_{l^{\prime}} \circ T^{i^{\prime}}-\sum_{l^{\prime}>l} g_{l^{\prime}} \circ T^{i}\right|}{\left(i^{\prime}-i\right)^{1 / 2-1 / p}} \neq 0\right\} & \\
\leqslant \sum_{l^{\prime}>l} \mu\left\{\max _{1 \leqslant i<i^{\prime} \leqslant N_{l}}\left|g_{l^{\prime}} \circ T^{i^{\prime}}-g_{l^{\prime}} \circ T^{i}\right| \neq 0\right\} & \leqslant N_{l} \sum_{l^{\prime}>l} \mu\left\{g_{l^{\prime}} \neq 0\right\},
\end{aligned}
$$

hence

$$
\begin{aligned}
\mu\left\{\frac{1}{N_{l}^{1 / p}} \max _{1 \leqslant i<i^{\prime} \leqslant N_{l}} \frac{\left|\sum_{l^{\prime}>l} g_{l^{\prime}} \circ T^{i^{\prime}}-\sum_{l^{\prime}>l} g_{l^{\prime}} \circ T^{i}\right|}{\left(i^{\prime}-i\right)^{1 / 2-1 / p}} \neq 0\right\} & \leqslant \\
& \leqslant 2 N_{l} \sum_{l^{\prime}>l} K_{l^{\prime}} / N_{l^{\prime}} .
\end{aligned}
$$

By (3.16) and (3.17), we get

$$
\begin{aligned}
\mu\left\{\max _{1 \leqslant i<i^{\prime} \leqslant N_{l}} \frac{\left|g \circ T^{i^{\prime}}-g \circ T^{i}\right|}{\left.\left(i^{\prime}-i\right)^{1 / 2-1 / p} \geqslant N_{l}^{1 / p} / 2\right\} \geqslant}\right. \\
\geqslant \mu\left\{\max _{1 \leqslant i<i^{\prime} \leqslant N_{l}} \frac{\left|\sum_{l^{\prime} \geqslant l}\left(g_{l^{\prime}} \circ T^{i^{\prime}}-g_{l^{\prime}} \circ T^{i}\right)\right|}{\left(i^{\prime}-i\right)^{1 / 2-1 / p}} \geqslant N_{l}^{1 / p}\right\} \\
\geqslant \mu\left\{\max _{1 \leqslant i<i^{\prime} \leqslant N_{l}} \frac{\left|\left(g_{l} \circ T^{i^{\prime}}-g_{l} \circ T^{i}\right)\right|}{\left(i^{\prime}-i\right)^{1 / 2-1 / p}} \geqslant N_{l}^{1 / p}\right\}-2 N_{l} \sum \sum_{l^{\prime}>l} K_{l^{\prime}} / N_{l^{\prime}} .
\end{aligned}
$$

Combining the previous inequality with (3.15), we obtain for each integer $l$,

$$
\mu\left\{\max _{1 \leqslant i<i^{\prime} \leqslant N_{l}} \frac{\left|g \circ T^{i^{\prime}}-g \circ T^{i}\right|}{\left(i^{\prime}-i\right)^{1 / 2-1 / p}} \geqslant N_{l}^{1 / p} / 2\right\} \geqslant \frac{1}{2}-\frac{K_{l}}{2 N_{l}}-2 N_{l} \sum_{l^{\prime}>l} K_{l^{\prime}} / N_{l^{\prime}},
$$

and by (3.11), the inequality

$$
\mu\left\{\max _{1 \leqslant i<i^{\prime} \leqslant N_{l}} \frac{\left|g \circ T^{i^{\prime}}-g \circ T^{i}\right|}{\left(i^{\prime}-i\right)^{1 / 2-1 / p}} \geqslant \frac{N_{l}^{1 / p}}{2}\right\} \geqslant \frac{1}{8}
$$

holds for $l$ large enough. We deduce that for such integers $l$ and each $\delta \in(0,1)$,

$$
\mu\left\{w_{1 / 2-1 / p}\left(\frac{1}{\sqrt{N_{l}}} S_{N_{l}}^{\mathrm{pl}}(g-g \circ T), \delta\right) \geqslant 1 / 2\right\} \geqslant \frac{1}{8},
$$

hence the process $\left(W_{n}(g-g \circ T)\right)_{n \geqslant 1}$ cannot be tight in $\mathcal{H}_{1 / 2-1 / p}[0,1]$.

It remains to show that the sequence $\left(n^{-1 / 2}\left(g-g \circ T^{n}\right)\right)_{n \geqslant 1}$ is bounded in $\mathbb{L}^{p}$. Notice that for a fixed integer $l \geqslant 1$, the equalities

$$
\left|g_{l}-g_{l} \circ T\right|=\left|h_{l}-h_{l} \circ T^{K_{l}}\right|=\frac{N_{l}^{1 / p}}{K_{l}^{1 / 2+1 / p}} \cdot \mathbf{1}\left(\bigcup_{j=1}^{2 K_{l}} T^{N_{l}-j} A_{l}\right)
$$


take place. This implies that

$$
\begin{aligned}
\left\|g_{l}-g_{l} \circ T\right\|_{p} & =\frac{N_{l}^{1 / p}}{K_{l}^{1 / 2+1 / p}} \cdot\left[\mu\left(\bigcup_{j=1}^{2 K_{l}} T^{N_{l}-j} A_{l}\right)\right]^{1 / p} \\
& \leqslant \frac{N_{l}^{1 / p}}{K_{l}^{1 / 2+1 / p}}\left(\frac{2 K_{l}}{N_{l}}\right)^{1 / p}=2^{1 / p} K_{l}^{-1 / 2}
\end{aligned}
$$

hence for each integer $n \geqslant 1,\left\|g_{l}-g_{l} \circ T^{n}\right\|_{p} \leqslant 2^{1 / p} n K_{l}^{-1 / 2}$. Let us define

$$
\widetilde{g_{l}}:=\frac{N_{l}^{1 / p}}{K_{l}^{1 / 2+1 / p}} \sum_{j=1}^{K_{l}} j \mathbf{1}\left(T^{N_{l}-j} A_{l}\right)
$$

If $K_{l} \leqslant n$, then

$$
\begin{aligned}
\widetilde{g}_{l}-\widetilde{g}_{l} \circ T^{n} & =\frac{N_{l}^{1 / p}}{K_{l}^{1 / 2+1 / p}}\left(\sum_{j=1}^{K_{l}} j \mathbf{1}\left(T^{N_{l}-j} A_{l}\right)-\sum_{j=1}^{K_{l}} j \mathbf{1}\left(T^{N_{l}-j-n} A_{l}\right)\right) \\
& =\frac{N_{l}^{1 / p}}{K_{l}^{1 / 2+1 / p}}\left(\sum_{j=1}^{K_{l}} j \mathbf{1}\left(T^{N_{l}-j} A_{l}\right)-\sum_{j=n+1}^{n+K_{l}}(j-n) \mathbf{1}\left(T^{N_{l}-j} A_{l}\right)\right)
\end{aligned}
$$

hence

$$
\left|\widetilde{g}_{l}-\widetilde{g}_{l} \circ T^{n}\right| \leqslant \frac{N_{l}^{1 / p}}{K_{l}^{1 / 2+1 / p}}\left(\sum_{j=1}^{K_{l}} j \mathbf{1}\left(T^{N_{l}-j} A_{l}\right)+\sum_{j=n+1}^{n+K_{l}}(j-n) \mathbf{1}\left(T^{N_{l}-j-n} A_{l}\right)\right),
$$

and the following upper bound follows:

$$
\mathbb{E}\left|\widetilde{g}_{l}-\widetilde{g}_{l} \circ T^{n}\right|^{p} \leqslant 2^{p-1} \sum_{j=1}^{K_{l}} j^{p} K_{l}^{-1-p / 2} \leqslant 2^{p-1} K_{l}^{p / 2}
$$

Treating in a similar manner the function $g_{l}-\widetilde{g}_{l}$, we observe that the following inequality holds:

$$
\left\|g_{l}-g_{l} \circ T^{n}\right\|_{p} \leqslant C_{p} \begin{cases}n K_{l}^{-1 / 2} & \text { if } K_{l}>n \\ K_{l}^{1 / 2} & \text { otherwise }\end{cases}
$$

where $C_{p}$ depends only on $p$ (neither on $n$, nor on $l$ ). For a fixed integer $n$, we denote by $i(n)$ the unique integer satisfying the inequalities $K_{i(n)} \leqslant n<K_{i(n)+1}$. 
By (3.21), we have

$$
\begin{aligned}
\left\|g-g \circ T^{n}\right\|_{p} & \leqslant \sum_{l=1}^{+\infty}\left\|g_{l}-g_{l} \circ T^{n}\right\|_{p} \\
& \leqslant C_{p}\left(\sum_{l=1}^{i(n)-1} K_{l}^{1 / 2}+K_{i(n)}^{1 / 2}+n K_{i(n)+1}^{-1 / 2}+\sum_{l=i(n)+2}^{+\infty} n K_{l}^{-1 / 2}\right) \\
& \leqslant 3 C_{p} \sqrt{n}+C_{p} \sqrt{n} \sum_{l=i(n)+1}^{+\infty} \frac{K_{l}}{K_{l+1}^{1 / 2}} \\
& \leqslant C_{p}\left(3+\sum_{l=1}^{+\infty} \frac{K_{l}}{K_{l+1}^{1 / 2}}\right) \sqrt{n}
\end{aligned}
$$

where we used (3.13) in the second inequality and condition (3.14) ensures finiteness of the right hand side in this inequality.

This concludes the proof of Theorem 2.6.

Acknowledgements. 1 The author would like to thank the referee for helpful comments which not only improved the presentation of the paper, but also the results of the initial version of Theorem 2.1 and Corollary 2.2.

The author also thanks Alfredas Račkauskas, Charles Suquet and Dalibor Volný for useful discussions and many valuable remarks and comments.

\section{A. Appendix}

For the reader's convenience, we state deviation inequalities for $\tau$-dependent and $\rho$-mixing sequences.

Notation A.1. If $\left(f \circ T^{j}\right)_{j \geqslant 0}$ is a (strictly stationary) sequence of random variables, we define

$$
s_{N}^{2}(f):=\sum_{i=1}^{N} \sum_{j=1}^{N}\left|\operatorname{Cov}\left(f \circ T^{i}, f \circ T^{j}\right)\right| .
$$

Notation A.2. Let $Y$ be an integrable random variable. We denote by $G_{Y}$ the generalized inverse of $x \mapsto \int_{0}^{x} Q_{Y}(u) \mathrm{d} u$.

The following Fuk-Nagaev inequality was established in Theorem 2 of [2].

Theorem A.3. Let $\left(f \circ T^{j}\right)_{j \geqslant 0}$ be a strictly stationary sequence of centered and square integrable random variables. Let $R:=\left((\tau / 2)^{-1} \circ G_{f}^{-1}\right) Q_{f}$ and $S=R^{-1}$. For any $\lambda>0$, any integer $N \geqslant 1$ and any $r \geqslant 1$,

$$
\mu\left\{\max _{1 \leqslant i \leqslant N}\left|S_{i}(f)\right| \geqslant 5 \lambda\right\} \leqslant 4\left(1+\frac{\lambda^{2}}{r s_{N}^{2}(f)}\right)^{-r / 2}+\frac{4 N}{\lambda} \int_{0}^{S(\lambda / r)} Q_{f}(u) \mathrm{d} u,
$$

and

$$
s_{N}^{2}(f) \leqslant 4 N \int_{0}^{\|f\|_{1}}(\tau / 2)^{-1}(u) Q_{f} \circ G_{f}(u) \mathrm{d} u .
$$

For $\rho$-mixing sequences, Shao (Theorem 1.2, [21]) showed the following inequality.

\footnotetext{
${ }^{1}$ The final publication is available at Springer via http://dx.doi.org/10.1007/s10959-015-0633-9
} 
Theorem A.4. Let $\left(f \circ T^{j}\right)_{j \geqslant 0}$ be a strictly stationary sequence of centered random variables and $q \geqslant 2$. Then there exists a constant $K$ depending only on $q$ and the sequence $(\rho(n))_{n \geqslant 1}$ such that for each integer $N$ and $x>0$,

$$
\mu\left\{\max _{1 \leqslant i \leqslant N}\left|S_{i}(f)\right| \geqslant x\right\} \leqslant N \mu\{|f| \geqslant A\}+
$$

$K x^{-q}\left(N^{q / 2} \exp \left(K \sum_{i=0}^{[\log N]} \rho\left(2^{i}\right)\right)\|f\|_{2}^{q}+N \exp \left(K \sum_{i=0}^{[\log N]} \rho^{2 / q}\left(2^{i}\right)\right)\|f \mathbf{1}\{|f| \leqslant A\}\|_{q}^{q}\right)$.

where A satisfies

$$
2 N \cdot \mathbb{E}[|f| \mathbf{1}\{|f| \geqslant A\}] \leqslant x .
$$

\section{REFERENCES}

[1] Patrick Billingsley, Probability and measure, third ed., Wiley Series in Probability and Mathematical Statistics, John Wiley \& Sons, Inc., New York, 1995, A Wiley-Interscience Publication. MR 1324786 (95k:60001)

[2] J. Dedecker and C. Prieur, Coupling for $\tau$-dependent sequences and applications, J. Theoret. Probab. 17 (2004), no. 4, 861-885. MR 2105738 (2005k:60059)

[3] Jérôme Dedecker and Paul Doukhan, A new covariance inequality and applications, Stochastic Process. Appl. 106 (2003), no. 1, 63-80. MR 1983043 (2004c:60111)

[4] Jérôme Dedecker and Clémentine Prieur, New dependence coefficients. Examples and applications to statistics, Probab. Theory Related Fields 132 (2005), no. 2, 203-236. MR 2199291 (2007b:62081)

[5] Jérôme Dedecker and Emmanuel Rio, On the functional central limit theorem for stationary processes, Ann. Inst. H. Poincaré Probab. Statist. 36 (2000), no. 1, 1-34. MR 1743095 (2001b:60032)

[6] Monroe D. Donsker, An invariance principle for certain probability limit theorems, Mem. Amer. Math. Soc., 1951 (1951), no. 6, 12. MR 0040613 (12,723a)

[7] P. Doukhan, P. Massart, and E. Rio, Invariance principles for absolutely regular empirical processes, Ann. Inst. H. Poincaré Probab. Statist. 31 (1995), no. 2, 393-427. MR 1324814 (96b:60083)

[8] D. Hamadouche, Invariance principles in Hölder spaces, Portugal. Math. 57 (2000), no. 2, 127-151. MR 1759810 (2001g:60075)

[9] H. O. Hirschfeld, A connection between correlation and contingency, Mathematical Proceedings of the Cambridge Philosophical Society 31 (1935), 520-524.

[10] A. N. Kolmogorov and Ju. A. Rozanov, On a strong mixing condition for stationary Gaussian processes, Teor. Verojatnost. i Primenen. 5 (1960), 222-227. MR 0133175 (24 \#A3009)

[11] John Lamperti, On convergence of stochastic processes, Trans. Amer. Math. Soc. 104 (1962), 430-435. MR 0143245 (26 \#804)

[12] Florence Merlevède, Magda Peligrad, and Sergey Utev, Recent advances in invariance principles for stationary sequences, Probab. Surv. 3 (2006), 1-36. MR 2206313 (2007a:60025)

[13] Thomas Mikosch and Alfredas Račkauskas, The limit distribution of the maximum increment of a random walk with regularly varying jump size distribution, Bernoulli 16 (2010), no. 4, 1016-1038. MR 2759167 (2012e:60097)

[14] Magda Peligrad, Invariance principles for mixing sequences of random variables, Ann. Probab. 10 (1982), no. 4, 968-981. MR 672297 (84c:60054)

[15] Alfredas Račkauskas and Charles Suquet, Necessary and sufficient condition for the Lamperti invariance principle, Teor. İmovīr. Mat. Stat. (2003), no. 68, 115-124. MR 2000642 (2004g:60050)

[16] Alfredas Račkauskas and Charles Suquet, Necessary and sufficient condition for the functional central limit theorem in Hölder spaces, J. Theoret. Probab. 17 (2004), no. 1, 221-243. MR 2054586 (2005b:60085)

[17] E. Rio, Théorie asymptotique des processus aléatoires faiblement dépendants, Mathématiques \& Applications (Berlin) [Mathematics \& Applications], vol. 31, Springer-Verlag, Berlin, 2000. MR 2117923 (2005k:60001) 
[18] M. Rosenblatt, A central limit theorem and a strong mixing condition, Proc. Nat. Acad. Sci. U. S. A. 42 (1956), 43-47. MR 0074711 (17,635b)

[19] R. J. Serfling, Moment inequalities for the maximum cumulative sum, Ann. Math. Statist. 41 (1970), 1227-1234. MR 0268938 (42 \#3835)

[20] Qi Man Shao, A remark on the invariance principle for $\rho$-mixing sequences of random variables, Chinese Ann. Math. Ser. A 9 (1988), no. 4, 409-412. MR 996917 (90f:60071)

[21] Maximal inequalities for partial sums of $\rho$-mixing sequences, Ann. Probab. 23 (1995), no. 2, 948-965. MR 1334179 (96d:60027)

[22] Ch. Suquet, Tightness in Schauder decomposable Banach spaces, Proceedings of the St. Petersburg Mathematical Society, Vol. V (Providence, RI), Amer. Math. Soc. Transl. Ser. 2, vol. 193, Amer. Math. Soc., 1999, pp. 201-224. MR 1736910 (2000k:60009)

[23] Dalibor Volný and Pavel Samek, On the invariance principle and the law of iterated logarithm for stationary processes, Mathematical physics and stochastic analysis (Lisbon, 1998), World Sci. Publ., River Edge, NJ, 2000, pp. 424-438. MR 1893125 (2003b:60049)

Université de Rouen, LMRS, Avenue de l’Université, BP 1276801 Saint-ÉtienneDu-Rouvray cedex, France.

E-mail address: davide.giraudo1@univ-rouen.fr 